\title{
A Novel Method for Coarse Iris Classification
}

\author{
Li Yu ${ }^{1}$, Kuanquan Wang ${ }^{1}$, and David Zhang ${ }^{2}$ \\ ${ }^{1}$ Department of Computer Science and Technology, \\ Harbin Institute of Technology, Harbin, 150001, China \\ \{1yu, wangkq\} @hit. edu.cn \\ ${ }^{2}$ Department of computing, The Hong Kong Polytechnic University, \\ Hung Hom, Kowloon, Hong Kong \\ csdzhang@ comp.polyu.edu.hk
}

\begin{abstract}
This paper proposes a novel method for the automatic coarse classification of iris images using a box-counting method to estimate the fractal dimensions of the iris. First, the iris image is segmented into sixteen blocks, eight belonging to an upper group and eight to a lower group. We then calculate the fractal dimension value of these image blocks and take the mean value of the fractal dimension as the upper and the lower group fractal dimensions. Finally all the iris images are classified into four categories in accordance with the upper and the lower group fractal dimensions. This classification method has been tested and evaluated on 872 iris cases and the accuracy is $94.61 \%$. When we allow for the border effect, the double threshold algorithm is $98.28 \%$ accurate.
\end{abstract}

\section{Introduction}

Biometrics is one of the most important and reliable methods for computer aided personal identification. The fingerprint is the most widely used biometric feature, but the most reliable feature is the iris and it is this that accounts for its use in identity management in government departments requiring high security.

The iris contains abundant textural information which is often extracted in current recognition methods. Daugman's method, based on phase analysis, encodes the iris texture pattern into a 256-byte iris code by using some 2-dimensional Gabor filters, and taking the Hamming distance [1] to match the iris code. Wildes [2], matches images using Laplacian pyramid multi-resolution algorithms and a Fisher classifier. Boles et al, extract iris features using a one-dimensional wavelet transform [3], but this method has been tested only on a small database. Ma et al. construct a bank of spatial filters whose kernels are suitable for use in iris recognition [4]. They have also developed a preliminary Gaussian-Hermite moments-based method which uses local intensity variations of the iris [5]. They recently proposed an improved method based on characterizing key local variations [6].

Although these methods all obtain good recognition results, all iris authentication methods require the input iris image to be matched against a large number of iris images in a database. This is very time consuming, especially as the iris databases being used in identity recognition growing ever larger. To reduce both the search time and computational complexity, it would be desirable to be able to classify an iris 
image before matching, so that the input iris is matched only with the irises in its corresponding category, but as yet the subject of iris classification has received little attention in the literature.

This paper is intended to contribute to the establishment of meaningful quantitative indexes. One such index can be established by using box-counting analysis to estimate the fractal dimensions of iris images with or without self-similarity. This allows us to classify the iris image into four categories according to their texture and structure.

\section{Counting Boxes to Estimate the Fractal Dimension of the Iris}

The concept of the fractal was first introduced by Mandelbrot [7], who used it as an indicator of surface roughness. The fractal dimension has been used in image classification to measure surface roughness where different natural scenes such as mountains, clouds, trees, and deserts generate different fractal dimensions. Of the wide variety of methods for estimating the fractal dimension that have so far been proposed, the box-counting method is one of the more used widely [8], as it can be computed automatically and can be applied to patterns with or without selfsimilarity.

In the box-counting method, an image measuring size $R \times R$ pixels is scaled down to $s \times s$, where $1<s \leq R / 2$, and $s$ is an integer. Then, $r=s / R$. The image is treated as a $3 D$ space, where two dimensions define the coordinates $(x, y)$ of the pixels and the third coordinate $(z)$ defines their grayscale values. The $(x, y)$ is partitioned into grids measuring $s \times s$. On each grid there is a column of boxes measuring $s \times s \times s$. If the minimum and the maximum grayscale levels in the $(i, j)^{\text {th }}$ grid fall into, respectively, the $k^{\text {th }}$ and $l^{\text {th }}$ boxes, the contribution of $n_{r}$ in the $(i, j)^{\text {th }}$ grid is defined as:

$$
n_{r}(i, j)=l-k+1
$$

In this method $N_{r}$ is defined as the summation of the contributions from all the grids that are located in a window of the image:

$$
N_{r}=\sum_{i, j} n_{r}(i, j)
$$

If $N_{r}$ is computed for different values of $r$, then the fractal dimension can be estimated as the slope of the line that best fits the points $\left(\log (1 / r), \log N_{r}\right)$.

The complete series of steps for calculating the fractal dimension are follows. First, the image is divided into regular meshes with a mesh size of $r$. We then count the number of square boxes that intersect with the image $N_{r}$. The number $N_{r}$ is dependent on the choice of $r$. We next select several size values and count the corresponding number $N_{r}$. Following this, we plot the slope $D$ formed by $\operatorname{plotting} \log \left(N_{r}\right)$ against $\log (1 / r)$. This indicates the degree of complexity, or the dimensions of the fractal. Finally, a straight line is fitted to the plotted points in the diagram using the 
least square method. In accordance with Mandelbrot's view, the linear regression equation used to estimate the fractal dimension is

$$
\log \left(N_{r}\right)=\log (K)+D \log (1 / r)
$$

where $K$ is a constant and $D$ denotes the dimensions of the fractal set.

\section{Iris Classification}

\subsection{The Calculation of the Fractal Dimension}

The calculation of the fractal dimension begins with preprocessing the original image to localize and normalize the iris. In our experiments, the preprocessed images were transformed into images measuring $256 \times 64$.

Because all iris images have a similar texture near the pupil, we do not use the upper part of the iris image when classifying an iris. Rather we make use only of the middle and lower part of the iris image. Preliminarily, we use the box-counting method to calculate the fractal dimension. To do this, we first divide a preprocessed iris image into sixteen regions. Eight regions are then drawn from the middle part of the iris image, as shown in Fig. 1. We call these the upper group. The remaining eight regions are drawn from the bottom part of iris image. These are referred to as the lower group. From these sixteen regions we obtain sixteen $32 \times 32$ image blocks. We then use the box-counting method to calculate the fractal dimensions of these image blocks. This produces sixteen fractal dimensions, $F D_{i}(i=1,2 \ldots 16)$. The mean values of the fractal dimensions of the two groups are taken as the upper and lower group fractal dimensions, respectively.

$$
F D_{\text {upper }}=\frac{\sum_{i=1}^{8} F D_{i}}{8}, \quad F D_{\text {lower }}=\frac{\sum_{i=9}^{16} F D_{i}}{8}
$$

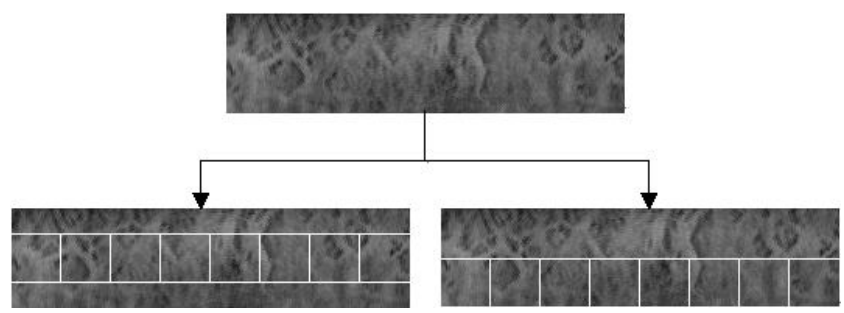

Fig. 1. Image segmentation

\subsection{Classifying an Iris Using the Double Threshold Algorithm}

The double threshold algorithm uses two thresholds to classify the iris into the following four categories, according to the values of the upper and lower group fractal dimensions. 
Category 1 (net structure): The iris image appears loose and fibrous. The fibers are open and coarse, and there are large gaps in the tissue. The values of both the upper and lower group fractal dimensions are less than the first threshold $E_{I}$.

$$
\left\{\left(F D_{\text {upper }}, F D_{\text {lower }}\right) \mid F D_{\text {upper }}<E_{I} \text { AND } F D_{\text {lower }}<E_{I}\right\}
$$

Category 2 (silky structure): The iris image appears silky. It displays few fibers and little surface topography. The Autonomic Nerve Wreath (also known as the Ruff and Collarette) is usually located less than one-third the distance from the pupil to the iris border. The values of the upper and lower group fractal dimensions are more than the second threshold $E_{I I}$.

$$
\left\{\left(F D_{\text {upper }}, F D_{\text {lower }}\right) \mid F D_{\text {upper }}<E_{I} \text { AND } F D_{\text {lower }}<E_{I}\right\}
$$

Category 3 (linen structure): The iris image appears to have a texture between those of Category 1 and Category 2. The Autonomic Nerve Wreath usually appears one-third to halfway between the pupil and the iris border, and the surface of ciliary zone is flat. (The Autonomic Nerve Wreath divides the iris into two zones, an inner pupillary zone, and an outer ciliary zone.) The value of lower group fractal dimension is more than the second threshold $E_{I I}$ and the value of upper group fractal dimension is less than the second threshold $E_{I I}$.

$$
\left\{\left(F D_{\text {upper }}, F D_{\text {lower }}\right) \mid F D_{\text {upper }}<E_{I} \text { AND } F D_{\text {lower }}<E_{I}\right\}
$$

Category 4 (hessian structure): The iris image appears to have a similar texture to Category 3 but with a few gaps (Lacunae) in the ciliary zone. When the upper and lower group fractal dimension values of an iris fail to satisfy the rules of Categories 1, 2 , or 3 , they are classified into Category 4.

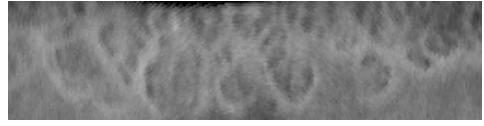

(a) Category 1

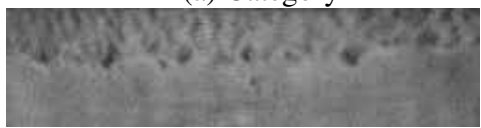

(c) Category 3

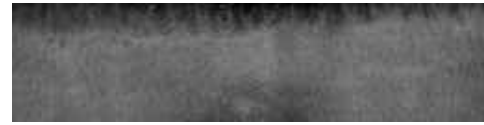

(b) Category 2

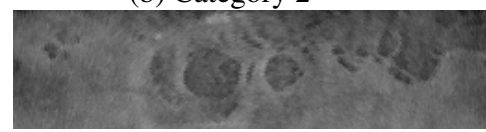

(d) Category 4

Fig. 2. Examples of each iris category after processing

Fig. 2 shows the range of possible textures. Categories 3 and 4 are both in a range between Categories 1 and 2. Category 3 is more like Category 2 and Category 4 is more like Category 1.Because the value of a fractal dimension is continuous, when classifying we must take into account the border effect. For the value near the threshold, we cannot simply classify the iris image into one category. Therefore, the nearby categories should be considered at the same time. The complementary rules for classifying the image are as follows: 
Rule 1. If $\left\{\left(F D_{\text {upper }}, F D_{\text {lower }}\right) \mid F D_{\text {upper }} \leq E_{I} \quad\right.$ AND $\left.\left.F D_{\text {lower }} \leq E_{I}+\Delta E\right)\right\}$ or $\left\{\left(F D_{\text {upper }}, F D_{\text {lower }}\right) \mid\left(E_{I}-\Delta E \leq F D_{\text {upper }} \leq E_{I}+\Delta E\right)\right.$ AND $\left.F D_{\text {lower }} \leq E_{I}\right\}$, the image belongs to Category 1 or Category 4, so Category 1 and Category 4 should be matched. Here $\Delta E$ is a small value.

$$
\begin{aligned}
& \text { Rule 2: If }\left\{\left(F D_{\text {upper }}, F D_{\text {lower }}\right) \mid\left(E_{I I}-\Delta E \leq F D_{\text {upper }} \leq E_{I I}+\Delta E\right) \quad\right. \text { AND } \\
& \left.E_{I I} \leq F D_{\text {lower }}\right\} \quad \text { or } \quad\left\{( F D _ { \text { upper } } , F D _ { \text { lower } } ) | E _ { I I } \leq F D _ { \text { upper } } \quad \text { AND } \quad \left(E_{I I}-\Delta E \leq\right.\right. \\
& \left.\left.F D_{\text {lower }} \leq E_{I I}+\Delta E\right)\right\} \text {, the image belongs to Category } 2 \text { or Category 3, so Category } 2 \\
& \text { and Category } 3 \text { should be matched. } \\
& \text { Rule } 3: \text { If }\left\{( F D _ { \text { upper } } , F D _ { \text { lower } } ) | F D _ { \text { upper } } < E _ { I I } - \Delta E \quad \text { AND } \quad \left(E_{I I}-\Delta E<\right.\right. \\
& \left.\left.F D_{\text {lower }}<E_{I I}+\Delta E\right)\right\} \text { the image belongs to Category } 3 \text { or Category 4, so Category } 3 \\
& \text { and Category } 4 \text { should be matched. }
\end{aligned}
$$

\section{Experimental Results}

Extensive experiments on a large image database were carried out to evaluate the effectiveness and accuracy of the proposed methods.

An iris image is correctly classified when the label of its category is the same as that of the iris. When there is no such match, the iris has been misclassified. The following subsections detail the experiments and their results.

Our iris classification algorithm was tested on a database containing 872 iris images captured from 436 different eyes. There are two images of each eye. The images measure $758 \times 568$ with eight bits per pixel and the irises have been labeled manually. Of the 872 irises in the database, 48 samples belong in Category 1, 336 belong in Category 2, 190 belong in Category 3 and 298 belong in Category 4.

After selecting the values for $E_{I}$ and $E_{I I}$, we carried out experiments on these two thresholds to classify the iris. Of the 872 irises in the database, 47 samples were misclassified: 6 in Category 1, 5 in Category 2, 20 in Category 3 and 16 in Category 4. This is a classification accuracy of approximately $94.61 \%$. Table 1 provides the confusion matrix. It shows that many misclassified irises are to be found in neighboring categories.

To reduce the influence of the border effect on classification accuracy, we have added three iris classification rules. If an iris satisfies one of the rules, it is simultaneously matched in two neighboring categories. Applying these rules, and with $\Delta E=0.0050$, the classification was $98.28 \%$ accurate. Clearly, this is a great improvement on the method which did not take into account the border effect.

Using coarse iris classification can reduce the time in searching. Table 2 shows the search time with and without coarse iris classification. As shown in Table 2, the search time of our iris recognition system can be reduced almost $70 \%$ of the original search time by using coarse iris classification, if taking into account of the border effect, the search time is less than half of the original search time. 
Table 1. Classification results of the double threshold algorithm

\begin{tabular}{ccccc}
\hline \multirow{2}{*}{$\begin{array}{c}\text { Assigned Category } \\
\text { No }\end{array}$} & \multicolumn{5}{c}{ True Category No. } \\
\cline { 2 - 5 } & 1 & 2 & 3 & 4 \\
\hline 1 & $\mathbf{4 8}$ & 0 & 0 & 6 \\
\hline 2 & 0 & $\mathbf{3 2 1}$ & 5 & 0 \\
\hline 3 & 0 & 9 & $\mathbf{1 7 5}$ & 11 \\
\hline 4 & 0 & 6 & 10 & $\mathbf{2 8 1}$ \\
\hline
\end{tabular}

Table 2. The search time of the system with and without coarse classification

without coarse classification(ms)
Using coarse classification

$$
\text { Without border effect (ms) Consider border effect (ms) }
$$

According to this rule, we can evaluate the size of the database to decide when to use the coarse classification method. Suppose $N$ is the database size, $T$ is the original search time without coarse classification and $T_{c}$ is the search time with coarse classification. The time used for iris coarse classification is $T_{f}$. If the computational cost for coarse classification is less than the reduced matching time, then this identification system can use coarse iris classification. That is:

$$
T_{f}<T-T_{c}
$$

As presented before, the reduced search time is about half of the original search time, So $T-T_{c}=T / 2$, and $T=N^{*} t$. Here $t$ is the match time (from a pair of feature vectors to the match result). Therefore, we obtained:

$$
T_{f}<N * t / 2
$$

In our method, $T_{f}$ and $t$ are about $98 \mathrm{~ms}$ and $0.2 \mathrm{~ms}$, so $N>980$. It shows that when the database size $N$ becomes bigger than 980 , the coarse classification can reduce the computational time of the identification system.

\section{Conclusion}

Among the biometrics approaches, iris recognition is known for its high reliability, but as databases grow ever larger, an approach needed that can reduce matching times. Iris classification can contribute to that. As the first attempt to classify iris images, this paper presents a novel iris classification algorithm based on the boxcounting method of fractal dimension. The approach uses the fractal dimension of the iris image to classify the iris image into four categories according to texture. The classification method has been tested and evaluated on 872 iris cases. After taking the border effect into account, the best result was obtained using the double threshold algorithm, which was $98.28 \%$ accurate. 
In the future, we will modify the image preprocessing method to reduce the influence of light and eyelids. There is also much work to be done on the selection of classification methods. We will also try other approaches to the improvement of classification accuracy.

\section{Acknowledgment}

This work is partially supported by $\mathrm{PhD}$ program foundation of the Ministry of Education of China, (20040213017), the central fund from The Foundation of the H.L.J Province for Scholars Return from Abroad (LC04C17) and the NSFC fund (90209020).

\section{References}

1. J.G. Daugman.: High Confidential Visual Recognition by Test of Statistical Independence. In: IEEE Trans. PAMI, Nov. vol.15, No.11, (1993) 1148-1161.

2. R. P. Wildes.: Iris Recognition: an Emerging Biometric Technology. In: Proc. IEEE, Sep. vol.85, (1997) 1348-1363.

3. W. W. Boles and B. Boashash.: A Human Identification Technique Using Images of the Iris and Wavelet Transform. In: IEEE Trans. Signal Processing, Apr, vol.46, No.4, (1998) 11851188.

4. L. Ma, T. Tan, Y. Wang and D. Zhang.: Personal Identification Based on Iris Texture Analysis. In: IEEE Trans. PAMI, Dec, vol.25, No.12, (2003) 1519-1533.

5. L. Ma, T. Tan, Y. Wang and D. Zhang.: Local Intensity Variation Analysis for Iris Recognition. In: Pattern Recognition, vol.37, (2004) 1287-1298.

6. L. Ma, T. Tan, Y. Wang and D. Zhang.: Efficient Iris Recognition by Characterizing Key local Variations. In: IEEE Trans. Image Processing, Jun, vol.13, No.6, (2004).739-749

7. B. B. Mandelbrot and J. W. Van Ness.: Fractional Brownian motions, fractional noises and applications. In: SIAM Rev., vol.10, no.4, (1968) 422-437.

8. H.O. Peitgen, H. Jurgens and D. Saupe.: Chaos and Fractals New Frontiers of Science. Berlin, Germany: Springer-Verlag, (1992) 202-213. 